\title{
Comment on "Reproducibility of Cerebrospinal Venous Blood Flow and Vessel Anatomy with the Use of Phase Contrast-Vastly Undersampled Isotropic Projection Reconstruction and Contrast-Enhanced MRA"
}

$I^{\mathrm{n}}$

n the introduction of the article "Reproducibility of Cerebrospinal Venous Blood Flow and Vessel Anatomy with the Use of Phase Contrast-Vastly Undersampled Isotropic Projection Reconstruction and Contrast-Enhanced MRA," ${ }^{1}$ the authors pointed out that the Zamboni hypothesis, known as chronic cerebrospinal venous insufficiency (CCSVI), "has prompted much interest in the accuracy and reproducibility of cerebrospinal venous flow measurements, which have not yet been established." The authors also stated that the diagnosis of CCSVI based on duplex (gray-scale and color Doppler) ultrasonography (US) has well-known limitations that can be overcome by using the MR imaging technique.

We agree with the authors about the need to investigate the cerebrospinal flow measurements, and we believe that MR imaging is a promising technique for this task. Nevertheless, the US technique is safe, allows real-time visualization of the moving structures, and is relatively inexpensive compared with MR imaging. Therefore, we are working to improve the US diagnostic methodology by using indexes based on the quantification of flow in the cerebrospinal veins to extend the current qualitative approach. ${ }^{2}$ Indeed, in our article, we proposed a simple model that, by assuming the conservation of mass criterion, allows us to work out the flow in the internal jugular veins (IJVs) and the collateral veins. One of our findings, also confirmed by Chambers et al, ${ }^{3}$ is that the flow increases as blood nears the heart. ${ }^{2,4}$ On the other hand, in Table 1 of their article, Schrauben et $\mathrm{al}^{1}$ reported that flow slightly decreases nearing the heart, by considering the left and right IJVs both as separated or as one. It is beyond the scope of

http://dx.doi.org/10.3174/ajnr.A3868 this letter to compare the potential of the 2 modalities in assessing cerebrospinal flow. Moreover, the number of subjects used in both studies is too low to draw conclusive results. In conclusion, we believe that at present, the US potential is not limited to a qualitative assessment of the cerebrospinal veins, and we recommend always comparing the MR imaging results with the US ones. This comparison will improve the knowledge of the cerebrospinal flow that, as the authors said, is still incomplete.

\section{REFERENCES}

1. Schrauben, EM, Johnson, KM, Huston, J, et al. Reproducibility of cerebrospinal venous blood flow and vessel anatomy with the use of phase contrast-vastly undersampled isotropic projection reconstruction and contrast-enhanced MRA. AJNR Am J Neuroradiol 2013 Nov 28. [Epub ahead of print]

2. Zamboni P, Sisini F, Menegatti E, et al. An ultrasound model to calculate the brain blood outflow through collateral vessels: a pilot study. BMC Neurol 2013;13:81

3. Chambers B, Chambers J, Churilov L, et al. Internal jugular and vertebral vein volume flow in patients with clinically isolated syndrome or mild multiple sclerosis and healthy controls: results from a prospective sonographer-blinded study. Phlebology 2013 Sep 24. [Epub ahead of print]

4. Zamboni P, Menegatti E, Pomidori L, et al, Does thoracic pump influence the cerebral venous return? J Appl Physiol 2012;112: 904-10

F. Sisini

M. Gambaccini

Department of Physics and Earth Sciences

E. Menegatti

P. Zamboni

Vascular Diseases Center

University of Ferrara

Cona, Italy 